\section{Infraoptic azygous anterior cerebral artery}

\author{
Sir,
}

A 22-year-old male presented with sudden headache and altered sensorium due to intracerebral hemorrhage [Figure 1A]. He had history of fever with elevated leukocyte counts. Echocardiogram showed valvular vegetations. DSA showed a mycotic aneurysm in the cortical branch of the right anterior cerebral artery (ACA). Infraoptic course of the azygous ACA was seen on the right side [Figure 1B and $\mathrm{C}$ ] with a hypoplastic left internal carotid artery (ICA) [Figure 1D, E and F]. Surgical clipping of the aneurysm as well as evacuation of the hematoma was done. Patient consciousness improved after the surgery and he started following verbal commands. He had significant hemiparesis at the time of discharge and hasn't come back for follow-up.

An infraoptic course of the ACA is rare, with approximately 30 previously reported cases..$^{[1,2]}$ Infraoptic ACA arises from the ICA at (or near) the origin of the ophthalmic artery instead of the usual site of origin at the ICA bifurcation. Angiography shows apparent low bifurcation of the ICA with the ACA arising near the ophthalmic artery which passes under the ipsilateral optic nerve before turning superiorly to join the normally positioned anterior communicating artery (AcomA). This anomaly may be detected on magnetic resonance (MR) angiography as a unusually low ICA bifurcation with the ACA ascending between the optic nerves ${ }^{[3]}$ It is most likely to be due to persistent in utero communication between the primitive dorsal and ventral ophthalmic arteries. ${ }^{[2]}$ Another theory favors persistence and enlargement of prechiasmatic anastomosis between the prechiasmal branch of the ophthalmic artery and chiasmal branches of the ACA..$^{[2]}$ It can be associated with agenesis of the ICA, anomalous origin of the ipsilateral ophthalmic artery from the external circulation, fused

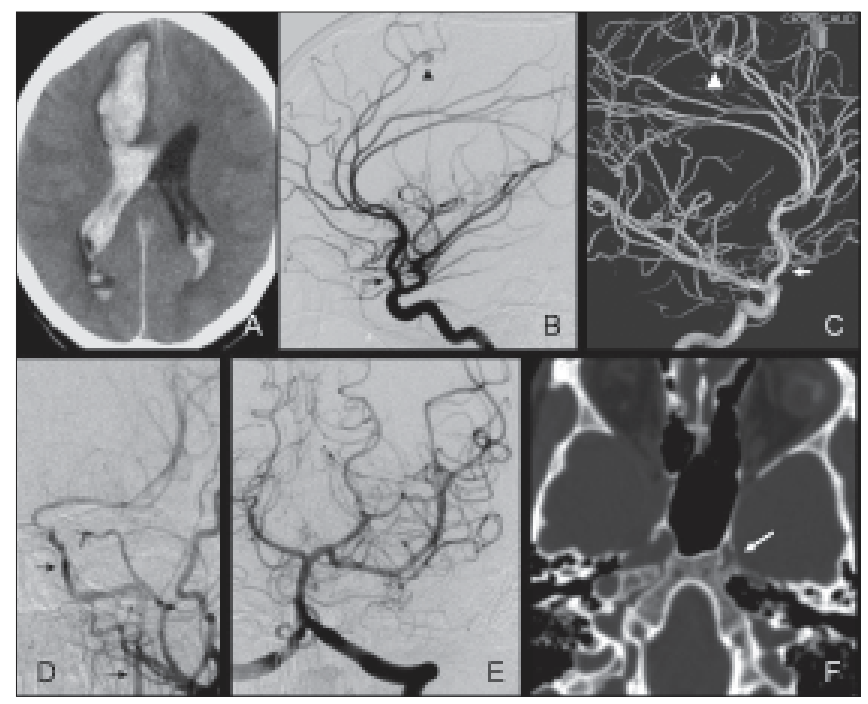

Figure 1: CT head shows right frontal hematoma with intraventricular extension (A). DSA (right ICA injection, lateral view) shows both ACA arising as a common trunk (azygous ACA) along with ophthalmic artery from paraclinoidal ICA (arrow, B). A mycotic aneurysm was seen in a cortical branch of right ACA (arrowhead, B). 3-D angiogram clearly delineates the infraoptic azygous ACA (arrow, C) and the aneurysm (arrowhead, C). Left common carotid injections reveal hypoplastic left ICA (arrows, D). Left vertebral artery injection shows left posterior communicating artery opacifying the middle cerebral artery. CT scan through base of skull shows hypoplastic left carotid canal, confirming congenital hypoplasia of left ICA (arrow, F)

pericallosal arteries, moyamoya disease, and symptoms related to the optic nerve or optic chiasma compression. ${ }^{[1]}$ To the best of our knowledge ours is the first reported case of azygous ACA associated with infraoptic ACA. Additionally, our case had an unusual anomaly of hypoplastic contralateral ICA associated with dilated posterior communicating artery providing collateral flow to the ICA territory. The prevalence of cerebral aneurysms associated with an infraoptic course of the ACA is much higher and the most common site is at the ACA-AcomA complex. ${ }^{[4]}$ Recognition of this anomaly is of considerable importance in planning surgery for ACA. ${ }^{[5]}$ In our case the distal location of the aneurysm presence of infraoptic ACA didn't result in alteration of surgical planning. However, in the event of temporary clipping of ACA, awareness of the azygous variant could have been relevant.

Vipul Gupta, Manish Chugh, Sandeep Vaishya ${ }^{1}$ Division of Interventional Neuroradiology and ${ }^{1}$ Neurosurgery, Max Institute of Neurosciences, Max Superspeciality Hospital, 1 Press Enclave Road, Saket, New Delhi-110 017, India. E-mail: vipulgupta25@yahoo.com

\section{References}

1. Given CA 2nd, Morris PP. Recognition and importance of an infraoptic anterior cerebral artery: Case report. AJNR Am J Neuroradiol 2002;23:452-4.

2. Spinnato S, Pasqualin A, Chioffi F, Da Pian R. Infraoptic course of the anterior cerebral artery associated with an anterior communicating 
artery aneurysm: Anatomic case report and embryological considerations. Neurosurgery1999;44:1315-9.

3. Given CA 2nd, Morris PP. Recognition and importance of an infraoptic anterior cerebral artery: case report. AJNR Am J Neuroradiol 2002;23:452-4.

4. Odake G. Carotid-anterior cerebral artery anastomosis with aneurysm: Case report and review of the literature. Neurosurgery 1988;23:654-8.

5. Senter HJ, Miller DJ. Interoptic course of the anterior cerebral artery associated with an anterior cerebral artery aneurysm: Case report. J Neurosurg 1982;56:302-4.

Accepted Date: 13-07-2008 